\title{
Virtual Labs in Electrical Engineering Education - The VEMA Environment
}

\author{
M. Travassos Valdez, \\ C. Machado Ferreira \\ Instituto Superior de Engenharia de \\ Coimbra \\ IPC/ISEC \\ Coimbra, Portugal \\ valdez@isec.pt
}

\author{
Maria João M. Martins \\ Academia Militar \\ DCTE \& INESC-ID \\ Lisboa, Portugal \\ mariajoaomartins2@gmail.com
}

\author{
F. P. Maciel Barbosa \\ Departamento de Engenharia \\ Eletrotécnica e de Computadores \\ Faculdade de Engenharia da Universidade \\ do Porto (FEUP) and INESC TEC \\ Porto, Portugal \\ fmb@fe.up.pt
}

\begin{abstract}
D worlds provide excellent platforms to achieve performance results when designed for the needs of students, helping them to improve acquisition and allowing greater interaction with contents in a unique way.

Virtual Labs are the future of education. Simulations, in which environmental measurements and data analysis can be simulated, are being created to combine real-world environments with information supplied by devices. Virtual Labs (VL) designed for educational purposes in Electrical Engineering provide tasks or problems within a virtual environment or context. Users can explore the environment and examine digital objects as they are designed to support inquiry-based learning and conceptual understanding.

Online virtual labs simulate learning environments and are designed to be highly attractive. They can complement the traditional formal learning programs, providing the opportunity for an interaction across a range of learning experiences in 3D. Online learning such as virtual classrooms will become the major part of training education in the future. Virtual lab applications are an engaging and agreeable learning system. Educational virtual labs should not merely focus on the virtual environment; they are useful to help students learn to think creatively and understand the complexities of solving meaningful problems, but the support from the teacher is also important.

This study presents a virtual scenario of an instrumentation and measurements laboratory. Students work with representations of electrical circuits focused on learning electrical concepts and circuit analysis, also studying and developing action procedures. The goal is to help them learn to think like engineers. While they are learning about circuit theory, they are also learning to apply the many skills of an engineer such as organizing information for writing technical reports.
\end{abstract}

This work was developed to demonstrate how a desktop Virtual Reality (VR) prototype, "Virtual Electric Manual"VEMA, can be applied to an engineering unit and used to enhance security and resourcefulness in using electrical equipment. Several interactive scenes were developed to illustrate the idea using a measurements and instrumentation laboratory as virtual environment.

Keywords-e-learning, interactive packages, virtual reality, 3D lab internet application, software

\section{INTRODUCTION}

Technology is crucial to integrate activities based teaching and learning methods. The type of software and hardware required is a matter of consideration as the technology involved should be easy for students and teachers to use and viable to set up. Different educational topics may require different technological solutions [1]. The implementation of educational technology can increase current learning, teaching and assessment processes by immersing students and teachers in an activity that is creative and pleasant for all. A carefully implemented activity that uses $3 \mathrm{D}$ visualization technology can improve current teaching and learning methods [2].

The use of simulations in teaching is becoming increasingly common. Simulations focus students on the essence of the problem, contributing to a more efficient assimilation of the topic viewed in each computer experiment. The use of simulators also allows students to investigate situations which may be demanding or even impossible to perform in a laboratory, thus providing a better and deeper understanding of the phenomena involved [3-7].

This work is the result of an investigation integrating the use of new technologies, especially the manipulation of computer simulations to teach selected topics of Circuit Theory. The choice of topics was based on the reported difficulty students have/feel when learning concepts related to these contents, and also due to the relevance of this subject, mainly its connection with natural phenomena and technology.

Technology alone cannot improve learning, as it is necessary to be smoothly integrated in activities based on teaching strategies.

The activities were devised based on an initial problem, its organization and use of previous learned concepts. The first activities present situations or equipment already familiar to the user.

It is necessary to discuss first the phenomenon and use of equipment to reach the specific concepts in order to organize the knowledge. Then, the concepts must be organized and extended to electrical mounts /situations as knowledge is applied.

The setup of activities is divided into two sets: one consisting of six general activities and the other one containing five simple circuits. Most of the activities consist of interactive simulations to build DC circuits (nine examples) and AC circuits (four examples). See Fig. 1. 


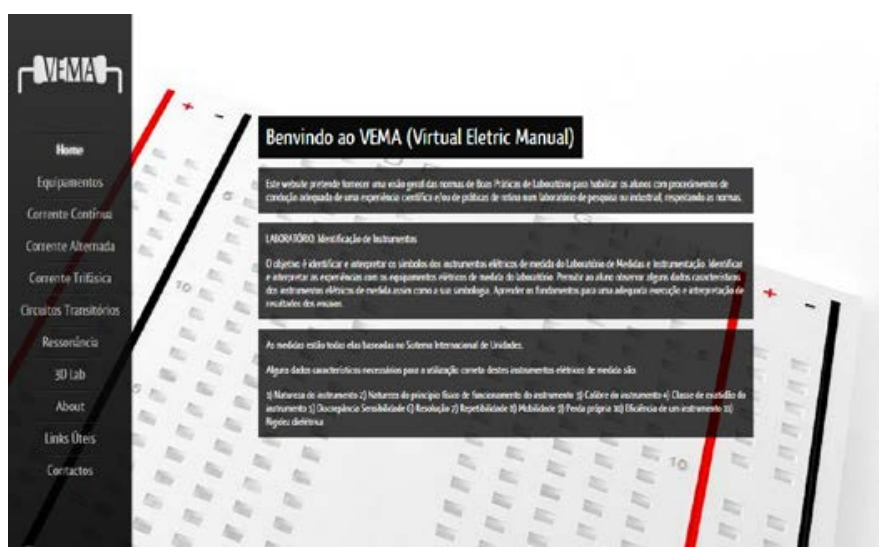

Fig. 1. First page of VEMA

\section{SimUlATIONS - EDUCATIONAL STRATEGIES}

The educational strategies of learning by doing and learning through practice are essential in this subject. The teaching and learning perspective is close to the constructivist theory of cognition. Students must learn a variety of techniques and skills necessary to carry out their projects. Learning is best achieved when they focus their work on solving problems defined in the short-term. Although the theory of constructivism is not new, technology can contribute to teaching and learning methods as theory can be applied more meaningfully [8]. With technology students can create their own understanding of the world and build their personal representation of knowledge. Learning occurs when they actively explore the knowledge leading to the discovery and there is interaction between students and their peers.

The concept of learning by doing is associated with the educational theory of experiential learning, in which the student is directly in touch with the realities being studied. In the learning process students absorb more when they take on a project or task and are allowed to make discoveries by themselves [9]. This involves questioning, analysis, experimentation, responsibility and the construction of meaning. This strategy allows learning to arise from natural significances, mistakes and successes, achieved by the students. By engaging in the action and reflecting on that same action, students achieve a deeper understanding of the theory and the practice involved. They are encouraged to develop the capacity for independent learning, solve problems in a creative way, identify and redefine problems. The purpose is to promote self-discovery, which can lead to a deeper approach to learning [10].

An approach based on research combines discoveries from previous findings and the testing prepares students to build up connections between real life phenomena and basic science, thus strengthening their understanding of the world they live in.

In order to further encourage quantitative exploration, simulations offer measurement instruments, including multimeters, power sources, oscilloscopes, voltmeters and function generators. As the user handles the interactive tools, the answers are immediately animated, showing cause-effect relationships as well as other representations (movement of objects, reading of data, etc.).

This way, students can use prior knowledge and skills to create new knowledge, and play a central role in controlling learning. With a lab simulation several perspectives of concepts can be presented with the focus on knowledge construction. At this point teachers are seen as guides and facilitators. All activities, tools and environments should help students to infer new knowledge/concepts, encouraging them to a deeper understanding and learning [10].
This approach aims at promoting learning and encouraging students to reflect not only on the subject, but also become autonomous learners.

The intention is to attract a large number of students and improve the quality of teaching and learning using new resources. With the advances of technology, and in particular the development of 3D virtual environments, new opportunities are presented, which may also solve some problems of lack of resources in some Institutions.

The need of a more autonomous and flexible learning environment led to the use of Virtual Learning Environments (VLEs), systems that provide access to online interactions of various kinds [7-8], [11-12].

\section{VIRTUAL LAB - VEMA}

One of the goals of VEMA was to create an attractive and motivating environment both for teachers and students. The prototype contains interactive simulations, virtual labs, testing as well as internet links which are called "learning objects". With a learning object of three, four or five minutes the teacher is able to present/explain a certain subject which could normally take him one or two 50 minutes sessions. The ability to create and explore 3D online environments presents several stimulating opportunities [10].

This kind of application can be integrated into the teaching and learning in a range of educational frameworks. It includes images, basic training guides and projects which students can use to practice.

VEMA may also be used in different levels of studies, ranging from basic learning, intermediate studies, and professional courses to young adults and adult education. Regarding virtual environments for teaching and learning, it seems to be quite positive.

VEMA (Virtual Eletrical MAnual), as an interactive application platform, aims at allowing students to explore and experiment several electrical experiments, without the need for being actually working in in the lab. The purpose is to provide another resource and support for higher education students to teach basic concepts in Circuit Theory. It enables any number of students, from any location, to use this simulated environment [13-14].

The prototype uses 3D visualization in which it is possible to manipulate contents, that is, change the position of an object, rotate it, pull it away, bring it forward, etc. The physical environment of a real lab is replicated, simulating workbenches or stored equipment. Some of the functions resemble a "game", allowing the student to choose one experiment within the range already prepared or create a whole new one.

All simulations are easy to handle and use in the classroom. They were built in 3ds Max, Vizup, Wirefusion or Java and may be run using any web server, with no need of any plugin installation.

The VR tool of this project uses a Wirefusion authoring tool, which allows a low cost and fast implementation when an educational license is used. This was a key factor in the choice of this specific application to implement VEMA. Moreover, Wirefusion does not generate 3D images or models; in turn, the developer creates a range of 3D objects, images, animations, 3D mesh and sounds in a separate program and then imports them to Wirefusion. The basic construction units in Wirefusion are called "objects". In short, an "object" is a reusable component containing a logical part of the application. This component can interact with the Wirefusion interface mechanism as well as other objects, and logical connections may be established among the different components in Wirefusion (Fig 2).

Wirefusion enables 3D models to be viewed online using any browser. VizUp reduces the size of 3D objects (too big to be easily functional). Java is used to control behaviors between the $3 \mathrm{D}$ elements and the interactions that the user performs. 


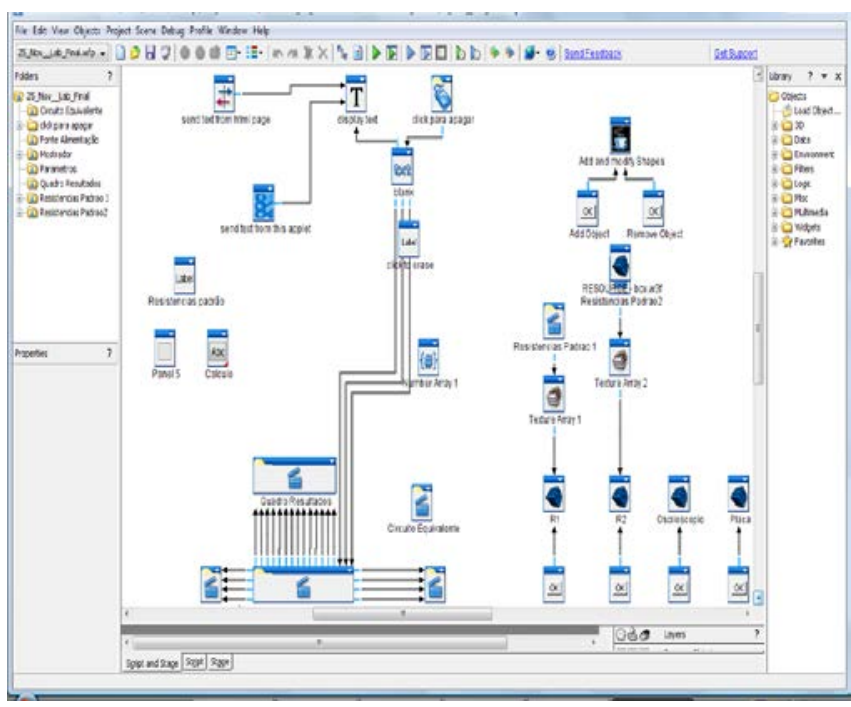

Fig. 2. Overall view of the Wirefusion user interface

The environment is based on 3D models and can be experienced in real-time. The student's view of the environment is rendered dynamically showing the actual position of objects in the virtual space. This system allows the student to move freely through the environment and the view is updated as he/she moves around.

All simulations were previously tested and assessed to ensure their efficiency and feasibility in an educational context. These tests included not only the simulations in a range of configurations, but also the actual lab tests.

Circuits with resistors and batteries can be built and data can be calculated using ammeters and voltmeters as if they were real.

Some of the learning objectives involve discussing basic electrical relationships; building circuits using schematics; using an ammeter and a voltmeter to take readings in circuits; providing arguments to explain the measurements and relationships in circuits; discussing basic electrical connections in series and parallel circuits; providing methods to explain the measurements in circuits.

The lab session should take no more than 30 minutes, including calculations and questions. Any activity in the lab is easy for the students to perform and easy to grade. It can be prepared at home with little instructor input if the student cannot attend the class. Students will go from station to station working with a different simulation to better visualize current, voltage and resistance. This provides a structure for a more detailed research work that facilitates acquisition and the interactive engagement.

Circuit Theory is stimulating when students are presented with familiar and relevant challenges. Electric circuits containing series, parallel and combination circuits are familiar to most students. The activity provides a real-life context to explore different kinds of circuits and their applications.

\section{A. Equipment}

There are eight different kinds of equipment for those who zap through the activity while others are still working (Fig. 3).

There is a set of simulations for the stage when students are introduced to current, voltage and resistance. The objective is to explain what voltage, resistance and current are and identify their roles in a circuit. The activity ends with the construction of circuits in series and in parallel both in the circuit simulation and in real life, using a real circuit board. The purpose is also to interpret and analyze how a circuit works and the different variables that affect the flow of energy.

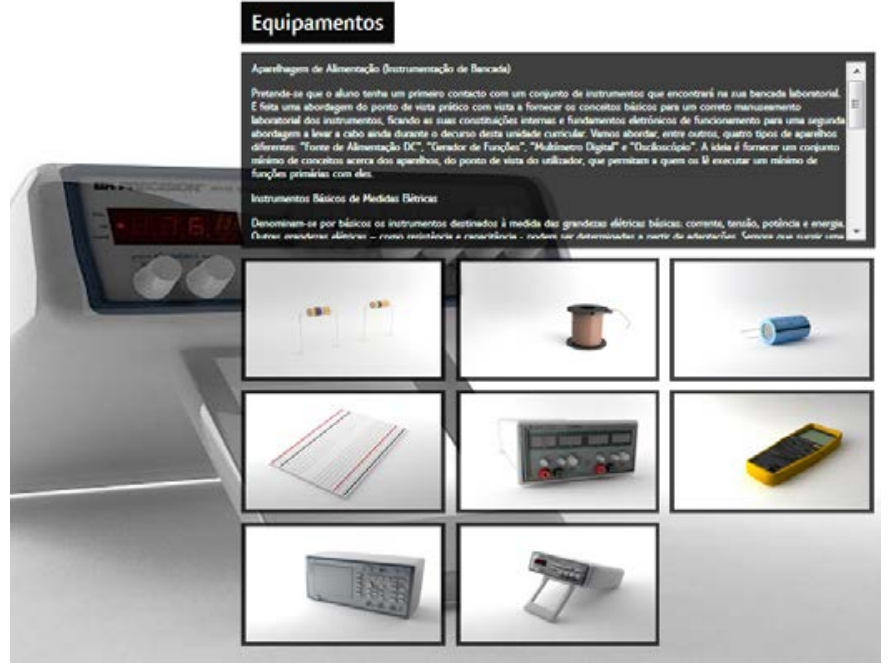

Fig. 3. VEMA Equipment menu

\section{B. Capacitor}

This menu allows to explore how a capacitor works and the measurement of voltage and of the electric field. The objectives will be: to determine the relationship between load and voltage for a capacitor as well as the energy stored in a capacitor or bank of capacitors in a circuit; also to explore the effect of added space and dielectric materials between the conductors of a capacitor in a circuit, and determine the equivalent capacity of a set of capacitors in series and in parallel in a circuit (Fig. 4).
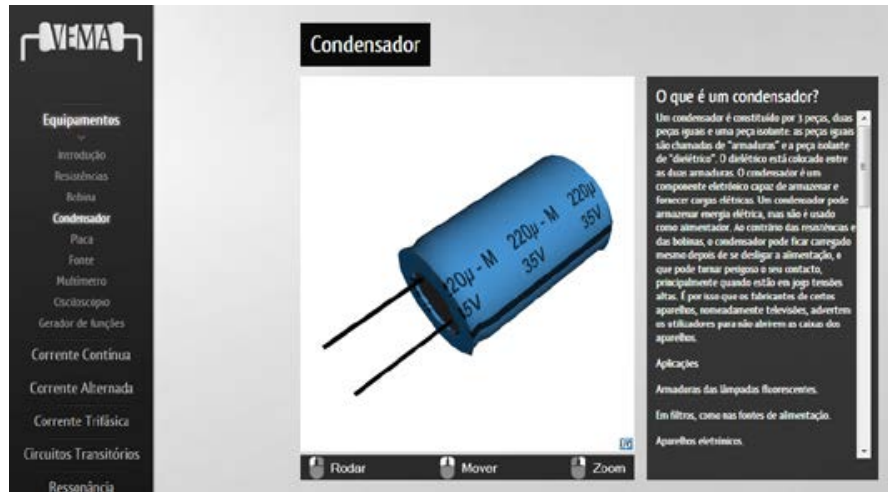

Fig. 4. Layout of the equipment

\section{Construction of DC circuits}

This menu presents a few simple DC circuits that will give an idea of how things work in the first place. Students will learn how to use a DC power supply and an electrician's best friend - a digital multimeter. They will understand the basic properties of circuits and what is required to complete a circuit. They will be able to build series and parallel circuits and understand the difference between the different circuits, and also learn about conductors and insulators.

This activity was used as an introduction to circuits (Fig. 5).

Students will use the circuit construction simulation to create circuits and verify Ohm's Law. Once they have been introduced to Ohm's law, they can use this simulation to help them visualize the concept of resistance and its relationship with voltage and current. They will go from simulation to simulation working with different ones to better visualize current, voltage and resistance. 


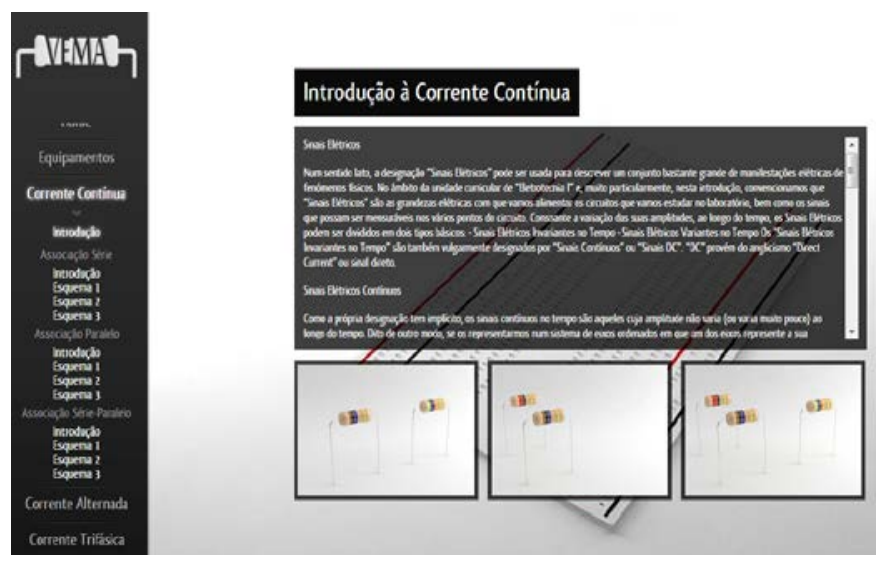

Fig. 5. VEMA - Direct Current, Introduction

This activity uses simulated circuits to allow students to investigate current, voltage and power relationships amongst the circuit elements for simple resistive circuits. It is intended for students to discover Kirchhoff's Loop Rule and Junction Rule by examining measurements made of nine different circuits, and to establish connections between energy and charge conservation with these rules. Power dissipated by each element is also examined and related with energy conservation.

Students collect and make a graph of data for changing voltage and resistance. This allows them to see different shapes of graph lines for direct and inverse relationships. Ohm's Law is explored. Current and Voltage are demonstrated to be directly proportional for a fixed resistance, and current and resistance are inversely proportional for a fixed voltage.

It is also necessary to show how to configure series and parallel circuits so they get a sense of what is happening inside a circuit as some of the various characteristics are altered.

In the first activity students explore the properties of circuits in series and in parallel made with identical resistors by using the DC circuit simulation. The second activity is hands-on and leads the students to set up circuits in parallel and in series with resistors of two different values. Voltages and currents are measured in several parts of the circuit to develop a general model for parallel and series circuits (Fig. 6).

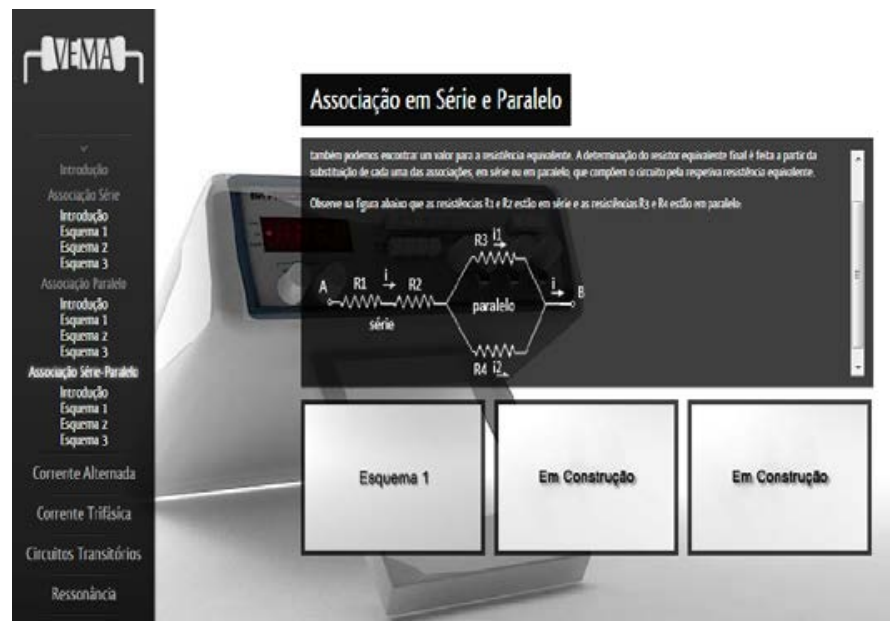

Fig. 6. VEMA - Direct Current, Series -Parallel Association

Students will be able to discuss basic electricity relationships in series and parallel circuits; analyze the differences between real circuits and the simulated ones; build circuits from schematic drawings; use a multimeter to take readings in circuits; provide reasoning to explain the measurements in circuits.

They will be able to use the simulation to learn the goals through discovery. This lab uses both simulation and lab equipment.

VEMA proposes to show the basic differences between series and parallel circuits. It walks students through the construction of series and parallel circuits using the simulators and asks them to record any observations. They will be able to build circuits to match the diagram to determine the relationship between voltage, current, and resistance in a series circuit, to understand series circuits electrical relationships.

They can explore the difference between circuits with batteries connected in series or parallel, learn how to relate the battery current to the current through resistors connected in parallel, and explore voltage in series circuits. Furthermore, they need to decide what they may want to place in series or in parallel. To discover the attributes of both they will use the computer simulation.

The objectives are: to place voltmeters and ammeters correctly in a series circuit; to find out the voltage and amperage in series circuits; and determine which is constant in a series circuit, voltage or amperage.

With resistance in parallel circuits the goals are to place voltmeters and ammeters correctly in a parallel circuit. Also; find the voltage and amperage in parallel circuits and determine which is constant in a parallel circuit, voltage or amperage. At the end, students are requested to build a virtual series or parallel circuit; measure voltage in parallel and series circuits; measure amperage in series and parallel circuits and compare the amperage and voltage in series versus parallel circuit.

These sessions are carried out after the preliminary concepts of series and parallel circuits are taught.

Combining series and parallel concepts into one circuit is the next step. Ideally, the students should attempt to solve the circuits first, before constructing them virtually. However, the simulator will provide a visual aid to help them understand how and why the current ends up splitting at each junction.

Most students found the use of simulation can become more useful and more enjoyable than the actual equipment. This simulation invites students to investigate the models shown to make sense, explain their ideas about the model, and describe their reasoning.

\section{AC Circuits}

A virtual lab experiment is used as a pre-lab activity or a replacement of a regular lab experiment. The student will be able to explain how a resistor, an inductor, or a capacitor affects the operation of an AC circuit (Fig. 7).
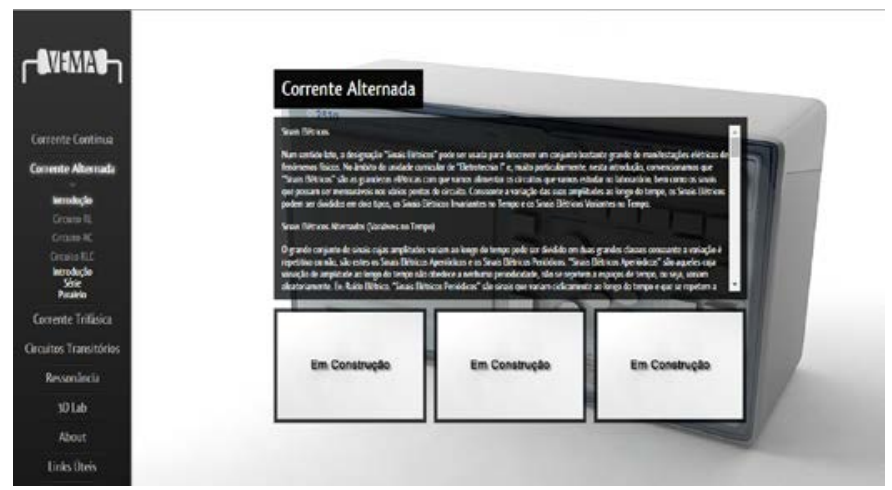

Fig. 7. VEMA - Alternating Current, Introduction and Series-Parallel Association 
This version of the simulation includes capacitors, inductors and AC voltage sources. The user can represent the current and the tension in a chart according to the time span.

The idea is to discuss the basic electrical relationships, build circuits from schematics, and use the ammeter and voltmeter to take readings in circuits. It will also be necessary to explain the measures and relationships in the circuits; discuss the basic relationships in series and parallel circuits or how a capacitor is loaded and discharged and discuss the behavior of an inductor in a circuit.

\section{E. Resonance}

This simulation will teach them how a radio tuning circuit works by investigating a circuit consisting of an inductor and a capacitor an LC circuit. When the activity is over, they should be able to describe the properties of a LC circuit; describe resonance in an LC circuit and why an LC circuit can be used as a radio tuner.

It is important to define the required conditions for a resonance; identify/explain the variables affecting the natural frequency of a system; identify which variables affect the duration of the transient behavior; explain that electrical resonance occurs in an electric circuit at a particular resonance frequency when the imaginary parts of impedances or admittances of circuit elements cancel each other; give examples of the application of real-world systems for which the understanding of resonance should be applied and explain why.

\section{CONCLUSION}

VEMA focused on a 3D learning environment with some embedded links. With VEMA students may compete with each other to design increasing complex circuits.

It provides the balance between the goals proposed by the simulation activity and the actual engagement of the simulation. This is the type of lab that invites students to get involved in simulations using models, allowing them to be able to reason and explain their ideas about the model based on the acquired virtual experience.

Virtual labs like VEMA offer very effective and engaging platforms, creating high-value learning exchanges, with the same sense of "presence" as in a real lab. The platform allows the creation of interactions that are both unique and meaningful for the student, including "hands on" and immersive experiences, unique to this environment.

VEMA provides an environment that helps students increase their security in circuit theory, providing asynchronous challenges such as picking up objects, or moving through the world to observe and participate in experiments. With VEMA, the 3D experiments allow various levels of learning, from cognitive to skills or competency based learning. It allows both passive and active learning, as well as synchronous and asynchronous learning. The costs associated to this platform are quite low when compared to other distance learning environments, and its power stands in the design and delivery. It also creates an environment where students can explore and discuss contents. VEMA can provide a blended approach, allowing students to learn on their own as well as interact in groups. VEMA provides real-time feedback of performance. It was also found that as students progressed through a learning program, their skill and confidence increased with each module, providing a consistent experience for various learning strategies. It was established that, by using sequencing modules over a period of time, students had the opportunity to practice concepts and skills, and were better prepared to discuss concepts in future sessions.

The VEMA environment can become a solution to Electrical Engineering Education as the virtual platform can bring training to students. It provides the experience of a face-to-face event without any inconvenience for the students who cannot attend classes. The
VEMA environment provides a new and effective learning delivery platform that can be used in a safe way. Learning through discovery by clicking on objects with associated information, simulating learning by modelling a process or interaction that closely resembles the real lab work in terms of fidelity and outcomes. It reinforces learning by offering aid menus, tools, etc., again associated with 3D objects. While learning with VEMA may not fully replicate the effectiveness of a face-to-face experiment, it is believed that it is worth the savings, risks and work efficiency gained through learning in $3 \mathrm{D}$. There is real value in designing effective learning for this $3 \mathrm{D}$ environment.

\section{REFERENCE}

[1] P. Ernest, M. G. Catasús, R. Hampel, S. Heiser, J. Hopkins, L.Murphy, U. Stickler, "Online teacher development: collaborating in a virtual learning environment”, Computer Assisted Language Learning, vol. 26, Issue 4, 2013.

[2] A. Ewais and O. De Troyer, "Authoring Adaptive 3D Virtual Learning Environments", International Journal of Virtual and Personal Learning Environments (IJVPLE) 5 (2014): 1, accessed July 10, 2014.

[3] R. M. Combs, and J. Mazur, "3D Modeling in a High School Computer Visualization Class: Enacting a Productive, Distributed Social Learning Environment." K-12 Education: Concepts, Methodologies, Tools, and Applications.IGI Global, 2014

[4] A. Nawaz, "Using e-learning as a tool for 'education for all' in developing states", International Journal of Science and Technology Educational Reseacrh, vol. 4(3), pp. 38-46, 2013. ISSN 2141-6559.

[5] K. Kreijns, F. Van Acker, M. Vermeulen, H. van Buuren, "What stimulates teachers to integrate ICT in their pedagogical practices? The use of digital learning materials in education", Computers in Human Behavior, vol. 29, Issue 1, January 2013, Elsevier, pp. 217-225, ISSN 0747-5632.

[6] P. Lacasa, "Learning in Real and Virtual Worlds: Commercial Video Games as Educational Tools", Palgrave Macmillan. ISBN: 978-1-13731204-4, September 2013. Accessed: 10 June 2014.

[7] P. Banyard, J. Underwood, L.Kerlin, J. Stiller, "Book Virtual learning environments: personalizing learning or managing learners?”, Palgrave Macmillan, chapter in Michael Thomas' (2011) book: "Digital Education: Opportunities for Social Collaboration". ISBN:9780230118003, 2011.

[8] B. Dalgarno and M. J. W. Lee, "What are the learning affordances of 3D virtual environments?”, British Journal of Educational Technology, Published by Blackwell Publishing Limited, 9600 Garsington Road, Oxford OX4 2DQ, UK, vol 41, No 1, pp. 10-32, 2010.

[9] D.A. Kolb, (1984), Experiential learning: experience as the source of learning and development, Englewood Cliffs, New Jersey, Prentice Hall.

[10] C. Stabile, and W. F. Ritchie, "Clarifying the Differences between Training, Development, and Enrichment: The Role of Institutional Belief Constructs in Creating the Purpose of Faculty Learning Initiatives”, New Directions for Teaching and Learning, 2013.

[11] N. Nussli and K. Oh, "The Components of Effective Teacher Training in the Use of Three-Dimensional Immersive Virtual Worlds for Learning and Instruction Purposes: A Literature Review", Journal of Technology and Teacher Education, vol. 22(2), pp. 213-241, 2014.

[12] J. Oerter, W. Suddarth, M. Morhardt, J. Gehringer, M. L. McGinnis, J. Shockley and A. Baysa, "A system architecture and simulation environment for building information modeling in virtual worlds", The Journal of Defense Modeling and Simulation: Applications, Methodology, Technology, July 2014.

[13] L. F. Gül, Ning Gu, A.Williams, "Virtual worlds as a constructivist learning platform: evaluations of 3D virtual worlds on design teaching and learning, ITcon vol. 13, Special Issue Virtual and Augmented Reality in Design and Construction , pp. 578-593, 2008.

[14] M. Travassos Valdez, C. Machado Ferreira and F. P. Maciel Barbosa, "Virtual Reality Prototype as a Tool in a Lab Environment". PRAXIS 2012, PRAXIS'12, The Open Discussion Forum. Wroclaw University of Technology. Poland. 6-7 September, 2012. 